Network Working Group

Request for Comments \#180

NIC \#7123

Categories: D.7, G.3

Updates: none

obsoletes: none
Alex McKenzie

BBN

25 June 1971

File System Questionnaire

As noted in RFC \#164 (page 35), a subcommittee of the NWG, under the chairmanship of Abhay Bhushan, is currently generating proposals for a "data transfer protocol" and a "file transfer protocol".

The subcommittee has decided that the file transfer protocol should provide standard methods for requesting the transfer of a file but should not, at this time, attempt to standardize file naming conventions, access control conventions, and the like. Thus a user who is, for example, trying to store a file on a remote Host will be required to use the file naming conventions appropriate to the remote Host.

Given the above point of view, it becomes imperative for users to have some source of information about Host file conventions. Such information, once compiled, will also serve as input to possible standardization efforts of the file transfer subcommittee. For this reason Abhay has asked me to solicit information on file conventions from the Host organizations. What follows is a description of the kinds of information of interest. I am well aware of the fact that many of you are tired of writing system descriptions; Xerox copies of short sections of your local documentation are fine if the result is complete and comprehensible. (In the case that your Host will never permit network use of your file system, a note to that effect would be sufficient.)

\title{
Information Requested
}

1. File naming conventions - We (loosely) define a pathname to be the data string which must be input to the file system by a user (a network user if your system makes a distinction between local and network users) in order to identify a file. We are interested in syntax, semantics, and defaults. Typical components of pathnames are:

\footnotetext{
- "device" fields

- user names

- version numbers

- index names

- punctuation marks
} 
Common types of defaults are:

- device is disk

- version number is largest in system

For hierarchical file structures, descriptions may be fairly complex, but with lots of defaults; in such cases an illustration of a "normal" pathname might be helpful.

2. Access control mechanisms - Access control mechanisms range from simply knowledge of a file's pathname to elaborate hierarchies of group-project-task-username membership with passwords and separate controls for reading and writing. There are two aspects of the access control mechanism which are of interest:

a. A description of what inputs the user should give the file system, both at the time of file creation and at the time of retrieval, in order to define the permitted modes of access and to gain access. What are the syntax and semantics of these inputs?

b. A description of the ways in which the access control mechanism is designed to help (or hinder) the sharing of files. For example, may two users "simultaniously" update a given file? May the creator of the file define a set of authorized users to the file system (and how)? Is it possible to define different access controls for various subunits of a given file?

3. Directories - Many systems maintain file directories which are designed to be helpful to the user. A directory might, for example, provide a list of all files created by a particular individual, along with some information regarding file size, file structure, access controls, etc. In general, such systems allow the user to input a pathname and retrieve the directory to which that pathname refers. Aspects of the directory structure of interest are:

a. What are the syntax and semantics of a directory pathname?

b. What use is a directory, i.e., what type of information does the directory contain?

c. What access controls are used for access to the directories? For example, must a user supply a password in order to retrieve a directory, and is this password typically the same as the password he would use to retrieve a file listed in that directory. 
4. Commands and functions of the file system - A general description of what the file system is designed to do would be useful. For example, the system might simply accept an entire file and store it sequentially on a tape; with the only mode of retrieval being to retrieve the entire file. On the other hand, the system might provide the ability to access any "subfield" with a unique pathname. Perhaps there is the ability to restructure a file, change the access control, delete all the fields associated with a directory, etc. We realize that this aspect of the file system begins to overlap the area of "data management", which is the responsibility of another subcommittee; therefore, use your judgement as to what functions are an intrinsic aspect of the file-handling system and which are aspects of "data-management".

5. Internal representation and I/O modes - The remote user of a file system will normally be interested in internal representation of data only insofar as that representation of data is reflected in the I/O interface between the file system and the network. For example, if all of the file system's I/O is in 8-bit ASCII characters, then the user is unlikely to care if they are stored in ASCII, EBCDIC, or some other form. However, if an alternate transmission mode is available it may be useful; for example, two PDP-10's, both of which store 5 characters and one "filler" bit per word, might find it advantageous to transfer information in this mode rather than converting between internal representation and 8-bit ASCII for each character. Other information on internal representation which would be of interest to the user might include (if applicable):

- range of numeric data permitted

- maximum text string lengths

- whether the user must indicate "record" boundaries on input

- what "logical structure" information the user may specify for a new file, and what he must specify

- whether the user must specify the file size before beginning input, and how he does it

6. Undoubtedly, there will be aspects of each file system which don't fit neatly into the categories above, but which users will find important or essantial in using the system. These should be identified and described if possible.

Please address responses to this questionnaire to:

Alex McKenzie

Bolt Beranek and Newman Inc.

50 Moulton Street

Cambridge, Massachusetts 02138

[Page 3] 
If the questions above are confusing, don't hesitate to call me for clarification at (617) 491-1850 ext. 441. I will issue another RFC summarizing the responses after I have received a significant number of them.

[ This RFC was put into machine readable form for entry ] [ into the online RFC archives by Stefan Hinker 6/97 ] 